\title{
Nucleotide composition of transposable elements likely contributes to AT/GC compositional homogeneity of teleost fish genomes
}

\author{
Radka Symonová ${ }^{* *}$ (i) and Alexander Suh ${ }^{2,3}$
}

\begin{abstract}
Background: Teleost fish genome size has been repeatedly demonstrated to positively correlate with the proportion of transposable elements (TEs). This finding might have far-reaching implications for our understanding of the evolution of nucleotide composition across vertebrates. Genomes of fish and amphibians are GC homogenous, with non-teleost gars being the single exception identified to date, whereas birds and mammals are AT/GC heterogeneous. The exact reason for this phenomenon remains controversial. Since TEs make up significant proportions of genomes and can quickly accumulate across genomes, they can potentially influence the host genome with their own GC content (GC\%). However, the GC\% of fish TEs has so far been neglected.

Results: The genomic proportion of TEs indeed correlates with genome size, although not as linearly as previously shown with fewer genomes, and GC\% negatively correlates with genome size in the 33 fish genome assemblies analysed here (excluding salmonids). GC\% of fish TE consensus sequences positively correlates with the corresponding genomic GC\% in 29 species tested. Likewise, the GC contents of the entire repetitive vs. nonrepetitive genomic fractions correlate positively in 54 fish species in Ensembl. However, among these fish species, there is also a wide variation in GC\% between the main groups of TEs. Class II DNA transposons, predominant TEs in fish genomes, are significantly GC-poorer than Class I retrotransposons. The AT/GC heterogeneous gar genome contains fewer Class II TEs, a situation similar to fugu with its extremely compact and also GC-enriched but AT/GC homogenous genome.

Conclusion: Our results reveal a previously overlooked correlation between GC\% of fish genomes and their TEs. This applies to both TE consensus sequences as well as the entire repetitive genomic fraction. On the other hand, there is a wide variation in GC\% across fish TE groups. These results raise the question whether GC\% of TEs evolves independently of GC\% of the host genome or whether it is driven by TE localization in the host genome. Answering these questions will help to understand how genomic GC\% is shaped over time. Long-term accumulation of GC-poor(er) Class II DNA transposons might indeed have influenced AT/GC homogenization of fish genomes and requires further investigation.
\end{abstract}

Keywords: Teleost fish, Transposon, GC content, Genome evolution, Nucleotide composition

\footnotetext{
* Correspondence: radka.symonova@gmail.com

'Department of Biology, Faculty of Science, University of Hradec Králové,

Hradec Králové, Czech Republic

Full list of author information is available at the end of the article
}

(c) The Author(s). 2019 Open Access This article is distributed under the terms of the Creative Commons Attribution 4.0 International License (http://creativecommons.org/licenses/by/4.0/), which permits unrestricted use, distribution, and reproduction in any medium, provided you give appropriate credit to the original author(s) and the source, provide a link to the Creative Commons license, and indicate if changes were made. The Creative Commons Public Domain Dedication waiver (http://creativecommons.org/publicdomain/zero/1.0/) applies to the data made available in this article, unless otherwise stated. 


\section{Background}

Nucleotide composition is a fundamental property of genomes with a strong influence on gene function and regulation [1]. Hence, GC content of a genome $\left(\mathrm{GC}_{\mathrm{G}}\right)$, i.e., the molar ratio of guanine $(\mathrm{G})$ and cytosine $(\mathrm{C})$ in $\mathrm{DNA}$, is one of the main parameters used to describe nucleotide composition and is frequently related to genome size [1]. For practical reasons, genomes can be segmented in five types of regions called isochores according to their GC percentage (GC\%). Two "light" isochores with the lowest GC\%, i.e., L1 with approx. 34$36 \%$ of GC and L2 approx. $37-40 \%$ of GC; as well as three "heavy" isochores, i.e., H1 with approx. $41-45 \%$ of GC, $\mathrm{H} 2 \quad 46-52 \%$ and the "heaviest" H3 with $>53 \%$ of GC [2]. In this regard, fish and amphibian genomes are overall AT/GC homogenous because they contain only the GC-poor(er) isochores with a substantially narrower range of $\mathrm{GC} \%$, i.e., usually only two neighbouring ones such as L1 and L2 or L2 and H1. On the other hand, avian and mammalian genomes contain all five isochores and their broad range of GC\% results in overall GC heterogeneity [2].

An increasing number of recent studies in fish has shown a clear positive correlation between genome size and percentage of TEs, and that TEs are ubiquitous and present in large numbers, e.g., refs. [3-6]. One of these studies [7] documented a surprisingly linear correlation between genome size and TE content in four teleost fish species. A clear but not strictly linear correlation between the percentage of TEs and genome size was identified in a larger dataset of 19 ray-finned and two lobe-finned fish species ([3]; including the four genomes analysed by ref. [7]). The so far most extensive (but still unpublished) study on fish TEs by ref. [5] using in silico explorations of TE activity, diversity and abundance across 74 teleost fish genomes showed that the total genomic TE abundances reflect variation in their host genome size.

Moreover, TEs can be very different in copy numbers and composition $[3,4,8,9]$, which would imply that accumulation or turnover of $\mathrm{TE}$ numbers/composition could change genomic $\mathrm{GC}$ content $\left(\mathrm{GC}_{\mathrm{G}}\right)$ because of the TEs' own $\mathrm{GC}$ content $\left(\mathrm{GC}_{\mathrm{TE}}\right)$. There are major quantitative and qualitative differences in TEs among vertebrates: Class II DNA transposons are the most abundant group in fish genomes, whereas in avian and mammalian genomes Class I retrotransposons are the most abundant group while DNA transposons are substantially less numerous $[3-5,8,9]$. Hence, the $\mathrm{GC}_{\mathrm{TE}}$ of different mobilomes, i.e., the sum of TEs within a genome, may potentially result in different overall $\mathrm{GC}_{\mathrm{G}}$ organization in fish when compared with birds and mammals. However, the characteristics of $\mathrm{GC}_{\mathrm{TE}}$ remains understudied in general, particularly in fish. This is despite the fact that TEs make up 6-55\% of the total base pairs of fish genomes, and that TEs are clearly depleted in compact and GC-rich genomes (Takifugu flavidus [9, 10], Tetraodon nigroviridis $[11,12]$ ) while they are massively represented in large and GC-poor genomes such zebrafish (Danio rerio [13]) and cod (Gadus morhua [14]).

The currently known main features of fish mobilomes can be summarized as follows: i. DNA transposons are the predominant group of TEs in fish; ii. the diversity of TE families is generally high in fish; iii. many TEs show recent activity in fish genomes; and iv. the total genomic abundances of TEs reflect the variation in genome size $[3-5,15]$. Since the dynamics of genome size variation can be largely explained by TEs in many eukaryotes $[16,17]$ and $G_{G}$ is negatively linked to genome size in some organisms [1], these findings in fish raise crucial questions about potential roles of TEs in shaping $\mathrm{GC}_{\mathrm{G}}$ : $\mathrm{i}$. Do TEs have a different $\mathrm{GC} \%$ than the non-TE regions of the host genome? ii. Do new TE insertions lead to a decrease in $\mathrm{GC} \%$ in adjacent regions of the host genome because of $\mathrm{TE}$ silencing through cytosine methylation? Methylcytosine frequently undergoes spontaneous deamination resulting in point mutation to thymine [18]. iii. Do TEs change local recombination rates (negatively if TEs are heterochromatinized or positively if they contain motifs attracting the recombination machinery $[19,20])$ and hence influence the $\mathrm{GC}_{\mathrm{G}}$ as discussed below? These factors all may contribute to the overall nucleotide compositional landscape, i.e., the heterogeneous organization in birds and mammals in comparison with the homogeneous organization in fish and amphibians. Such manifold effects of TEs might be particularly pronounced in species where TEs comprise a substantial genomic fraction, e.g., zebrafish (D. rerio) [13].

Both the local $\mathrm{GC}_{\mathrm{G}}$ as well as $\mathrm{TE}$ density are linked to the local recombination rate. Evidence to date suggests that TE densities correlate negatively with recombination rate, but the strength of this correlation varies across TE types [20]. At the same time, the currently most plausible explanation of the AT/GC heterogeneity in avian and mammalian genomes is a non-adaptive process called GC-biased gene conversion (gBGC), whereby increased GC\% is tightly related to an increased recombination rate (recently extensively reviewed by ref. [19]). In mammals and some other vertebrates (but not birds), at least a part of the regional variation in the location of recombination hotspots can be ascribed to the activity of the protein PRDM9 [21].

One may expect that TEs contribute to the length and GC\% of noncoding sequences, and continue to do so even long after they are no longer recognizable as TEs. While TE insertions are a major factor in the expansion or turnover of noncoding regions (both introns and 
intergenic sequences $[17,22])$, the potential influence of the $\mathrm{GC}_{\mathrm{TE}}$ on the host regional $\mathrm{GC}_{\mathrm{G}}$ has only been comprehensively assessed for the human genome. Around $42 \%$ of the human genome is made up of retrotransposons, whereas DNA transposons only account for about $2-3 \%$, and the insertion or accumulation of TEs depends on the isochore region involved [23]. For instance, Alu (the most abundant TE in human) and L1 insertions contribute to the AT/GC heterogeneity of the human genome due to their differential accumulation: Alu SINEs (approx. $50 \% \mathrm{GC}_{\mathrm{TE}}$ in their consensus sequence) reside preferentially in GC-rich regions, whereas L1 LINEs (approx. 37\% $\mathrm{GC}_{\mathrm{TE}}$ in their consensus sequence) reside preferentially in GC-poor regions [24]. Recognizable Alu elements make up $20 \%$ of GC-rich regions and $7 \%$ of GCpoor regions, whereas recognizable $\mathrm{L} 1$ elements make up $5 \%$ of GC-rich regions and 20\% of GC-poor regions [25]. For fish, a single study briefly investigated the potential correlation between TEs and GC\% along T. nigroviridis and $D$. rerio genomes [26]. However, they did not observe any effect of TEs on $\mathrm{GC}_{\mathrm{G}}$ in $T$. nigroviridis and $D$. rerio. Three studies investigated in detail some unusual examples of GC-rich TEs in crabs [27-29] and reported different GC\% between DNA transposons of marine and continental species. A bit more is known from plants and their TEs, e.g., Pack-MULEs elements in grasses specifically acquire and amplify GC-rich gene fragments [30].

In this study, we aim to bring a novel viewpoint on the vertebrate nucleotide compositional evolution by analysing the $\mathrm{GC}_{\mathrm{TE}}$ of fish TEs and assessing their potential contribution to the $\mathrm{GC}_{\mathrm{G}}$ and the overall nucleotide compositional landscape of their host genomes.

\section{Results}

\section{Genome size positively correlates with the genomic} density of TEs in fish

To summarize the previously reported positive correlation between fish genome size and genomic abundance of TEs $[3-5,7,15]$, we generated an example plot using cytological genome size estimates, i.e. Cvalue in picograms (pg; Fig. 1a). Species included are 29 teleosts that underwent the teleost-specific wholegenome duplication (WGD) of which five salmonid species underwent another round of WGD, the salmonid-specific one [35]. Further, we included the spotted gar (Lepisosteus oculatus), i.e., a deepbranching non-teleost ray-finned fish that has not undergone any further WGD after the two basal vertebrate ones but that shows the mammalian-like situation of AT/GC heterogeneity [36]. Finally, we analysed one lamprey species (Petromyzon marinus), one shark (Callorhinchus milii) and one coelacanth (Latimeria chalumnae). This correlation represents an important starting point for our following considerations. Detailed lists of species analysed are in Additional files 1 and 2: Tables S1 and S2.

\section{Genome size negatively correlates with the genomic GC\% in fish excluding salmonids}

Data on $\mathrm{GC}_{\mathrm{G}}$ of genome assemblies currently available in NCBI GenBank [33] and in the literature permit us to identify another crucial association - a negative correlation between fish genome size (as $\mathrm{C}$-value in picograms from the Animal Genome Size Database [32]) and their genomic GC\% (Fig. 1b).

To avoid any potential bias conditioned by incompleteness of currently available genome assemblies (e.g., differences in amounts of heterochromatic repeats assembled and in assembly quality sensu [37]), we compared two types of genome size datasets: one based on $\mathrm{C}$-values, i.e., the non-genomics (cytological) genome size estimation (Fig. 1b) and another based on genome assembly size (Fig. 1d). Despite slight differences between these datasets, both show comparable trends, suggesting that both are usable for further analyses.

In this analysis, we excluded the eight sampled salmonid species (details in Additional file 1: Table S1) because their large genomes exhibit a salmonid-specific WGD and extremely amplified ribosomal (rRNA) genes that are exceptionally GC-rich. This feature is well known from cytogenetics [31]. Including these large and GC-enrich salmonid genomes distorts the clear correlation between $\mathrm{GC}_{\mathrm{G}}$ and genome size in other teleost fish (cf. Additional file 3: Figure S1).

\section{GC\% of TEs positively correlates with genomic GC\% in fish}

Comparison of $\mathrm{GC}_{\mathrm{TE}}$ with the respective $\mathrm{GC}_{\mathrm{G}}$ uncovered a positive correlation. Firstly, we calculated the $\mathrm{GC}_{\mathrm{TE}}$ out of the sum of individual consensus sequences of TEs annotated for each fish species from FishTEDB [34] (Fig. 1c) and not out of the entire mobilome reflecting the TEs' copy numbers in the respective genome. As consensus sequences are approximations of the TE copies at their time point of insertion, we consider their consensus $\mathrm{GC}_{\mathrm{TE}}$ to be more appropriate here because it should not reflect the genomic location of individual TE copies. Note that FishTEDB does not include any salmonid species. For comparison, we calculated $\mathrm{GC}_{\mathrm{REP}}$ of repeats including low-complexity regions and compared it with the remaining non-repetitive fraction of the relevant genomes, i.e. $\mathrm{GC}_{\text {NONREP }}$ (Fig. 2). For this analysis, we used masked genome assemblies from the Ensembl (Release 98, [38]) as the FishTEDB lists only consensus sequences of TEs per fish species.

The $\mathrm{GC}_{\mathrm{TE}}$ is mostly higher than the overall $\mathrm{GC}_{\mathrm{G}}$, with two exceptions. These exceptions are cod and European 

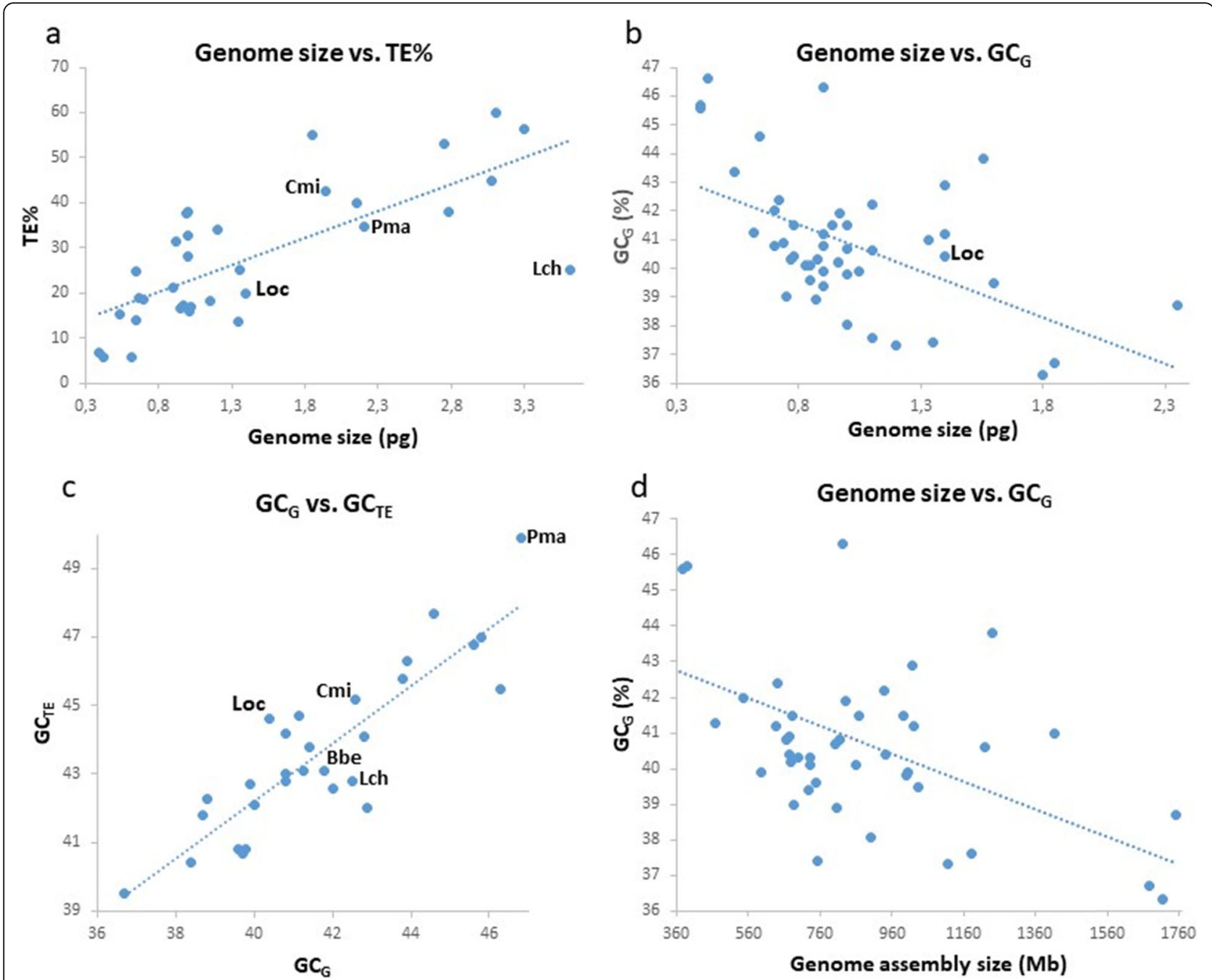

Fig. 1 Genome size, transposable elements, and nucleotide composition. a Abundance of transposable elements in 29 teleosts, one non-teleost ray-finned fish (spotted gar, L. oculatus; Loc) with a AT/GC heterogeneous genome, one lobe-finned fish (L. chalumnae; LCh), one lamprey ( $P$. marinus; Pma) and one shark (C. milii; $\mathrm{Cmi}$ ) species related to their host genome size (genome size as C-value in picograms, pg), data from [3]. b GC percentage (GC\%) of 46 fish genomes with available genome assemblies (excluding salmonids with their rediploidized genomes exceptionally enriched in extremely GC-rich rRNA genes [31]) negatively correlates with fish genome size based on averaged cytological measurements (Cvalue in pg, multiple C-value records were averaged). C-value data from the Animal Genome Size Database [32], GC\% data from GenBank [33]. c GC\% of TE consensus sequences (not accounting for their copy number within genomes) positively correlates with the overall GC\% of the host genome in 25 ray-finned fish species, one lancelet (Branchiostoma belcheri; Bbe), one lamprey (Pma), one shark (Cmi) and one coelacanth included in FishTEDB [34]. Genomic GC\% data are from GenBank [33], GC\% of TEs was calculated from species-specific TE consensus sequence libraries from FishTEDB [34]. d GC\% of genome assemblies (in Mb) of 58 fish species listed GenBank [33]

eel, however, the difference is within the range of $1 \%$, i.e., for the eel $\mathrm{GC}_{\mathrm{G}}=42.9 \%$ vs. $\mathrm{GC}_{\mathrm{TE}}=42.0 \%$ and for the cod $\mathrm{GC}_{\mathrm{G}}=46.3 \%$ vs. $\mathrm{GC}_{\mathrm{TE}}=45.5 \%$ (more details in Additional file 4: Figure S2).

GC\% varies widely among particular groups of TEs in fish Dissecting the GC anatomy of the sum of individual $\mathrm{TE}$ consensus sequences in fish genomes, we further disentangled $\mathrm{GC}_{\mathrm{TE}}$ of the major TE groups: Class I retrotransposons are GC-richer with an averaged consensus $\mathrm{GC}_{\mathrm{TE}}$ of $45.6 \%$ than Class II DNA transposons with an averaged consensus $\mathrm{GC}_{\mathrm{TE}}$ of $40.1 \%$ (Fig. 3). Within Class I, LTR retrotransposons are GC-richer than LINEs. The Class I DIRS retrotransposons are the GC-richest fish TEs with $\mathrm{GC}_{\mathrm{TE}}$ of $53.8 \%$. The Class II CMC transposons are the AT-richest fish TEs with $\mathrm{GC}_{\mathrm{TE}}$ of $35.8 \%$.

Details on the variability of species-specific $\mathrm{GC}_{\mathrm{TE}}$ in 19 selected species from FishTEDB are presented in Figure S3 (Additional file 5; 16 ray-finned species, one lancelet, one shark, and one lamprey species; some species displayed in FishTEDB do not contain sequences). 


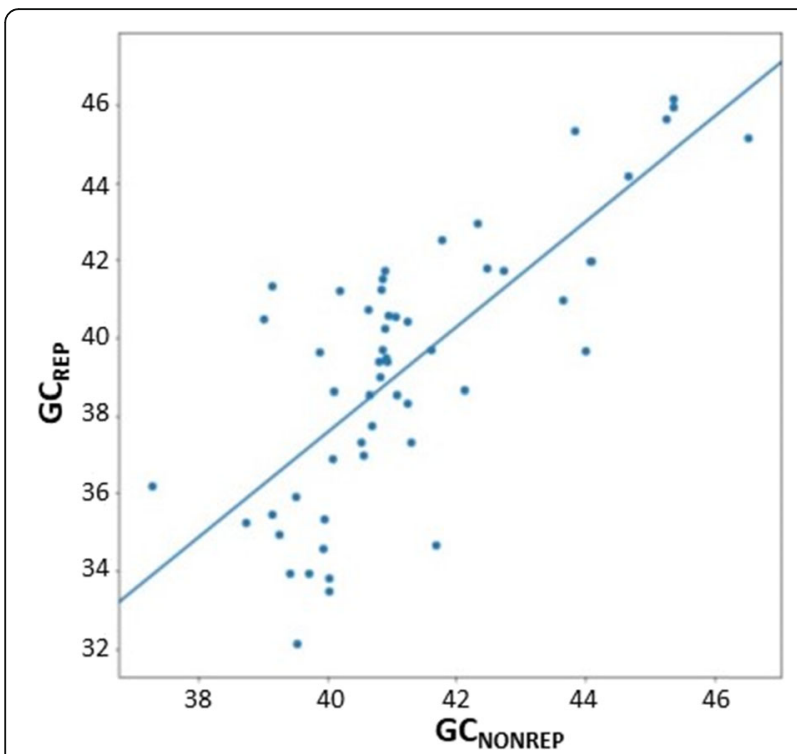

Fig. 2 Comparison of GC\% of repetitive and non-repetitive genomic fractions in 54 fish species from the Ensembl database (Release 98). The $Y$-axis shows $\mathrm{GC}_{\text {REP }}$, i.e. $\mathrm{GC} \%$ of repeats (including lowcomplexity regions) masked with the RepeatMasker tool, while the $X$-axis shows $\mathrm{GC}_{\text {NONREP }}$ of the non-repetitive fraction of each assembly. Data used for this analysis are available in the Additional file 2: Table S2

\section{GC\% of Class II DNA transposons varies heavily among}

\section{different fish species}

The observed variation in $\mathrm{GC}_{\mathrm{TE}}$ among the major $\mathrm{TE}$ groups listed in the FishTEDB is particularly relevant considering that fish genomes are greatly enriched in Class II DNA transposons in contrast to avian and mammalian genomes. Therefore, we calculated the $\mathrm{GC}_{\mathrm{TE}}$ of all consensus sequences of DNA transposons for 17 fish species. These data provide first insights into the $\mathrm{GC}_{\mathrm{TE}}$ of fish transposons. Firstly, the compact genomes of not only pufferfishes $T$. flavidus and T. nigroviridis but also of $\operatorname{cod}(G$. morhua) and stickleback (Gasterosteus aculeatus) show GC enrichment of their TEs as well as overall GC-richer Class II DNA transposons (Fig. 4). The same is apparent also in the non-teleost spotted gar (L. oculatus) with its AT/GC heterogeneous genome and an unusually high $\mathrm{GC}_{\mathrm{TE}}$ in comparison with teleosts. The opposite situation occurs in teleosts with larger genomes such as D. rerio and Astyanax mexicanus: DNA transposons are $\mathrm{GC}$-poor(er) as well as the overall $\mathrm{GC}_{\mathrm{G}}$ and $\mathrm{GC}_{\mathrm{TE}}$ are lower.

\section{Discussion}

Recent studies on the relative contribution of TEs to genome size in fish $[3,4,7,39]$ have become an important starting point for us to understand the evolution of nucleotide composition. The above listed results raise crucial questions about the contribution of the mobilome $\mathrm{GC} \%$ to the entire genomic $\mathrm{GC} \%$ and to the nucleotide compositional landscape. This has been so far addressed only for the human genome [22]. Here, we show that utilizing purely genomic data for approximating genome size (assembly vs. C-value) and GC\% yield reproducible and comparable data suitable for assessing nucleotide composition of host genomes and their respective TEs. The ever-increasing number of available assemblies and TE annotations for fish and other

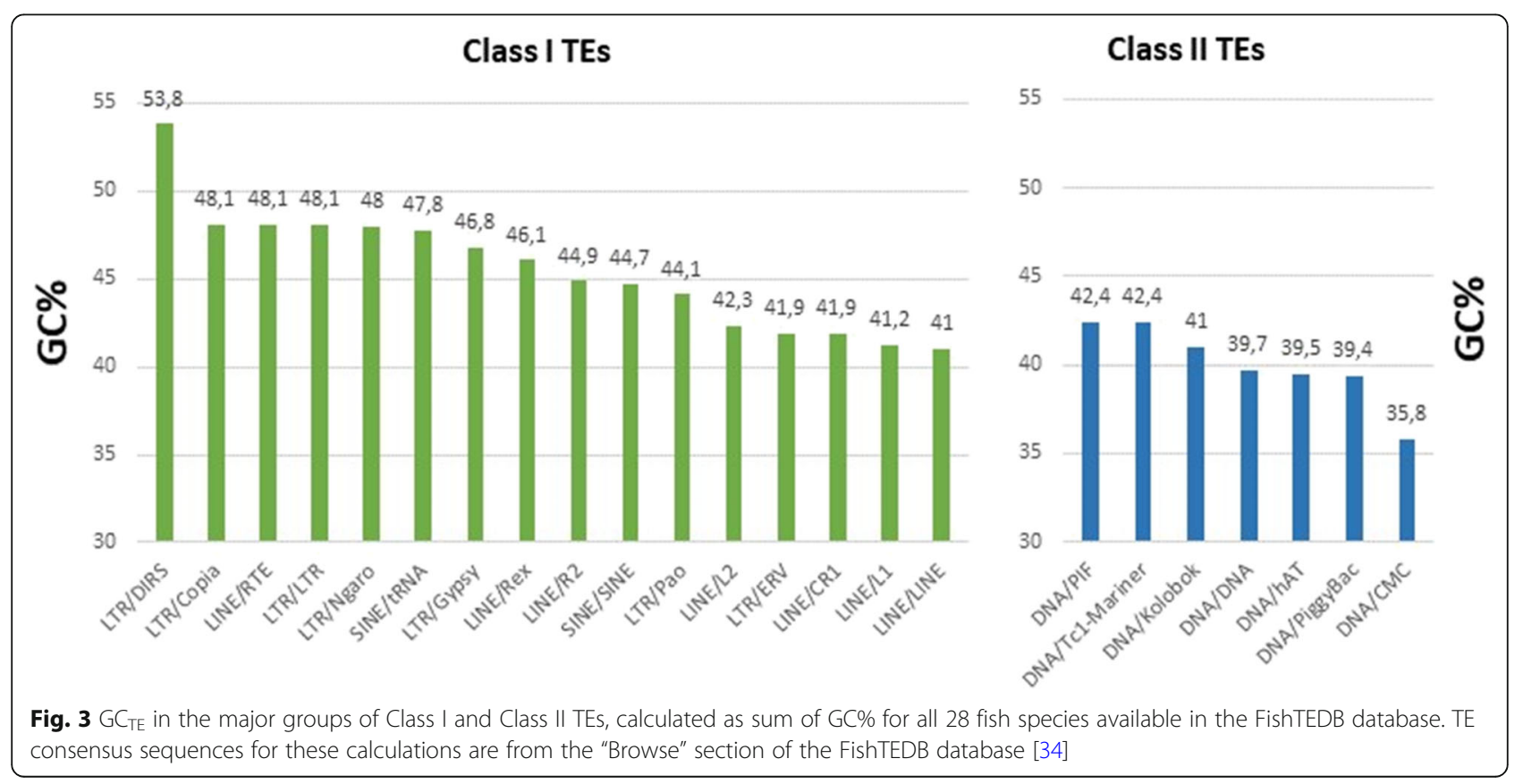




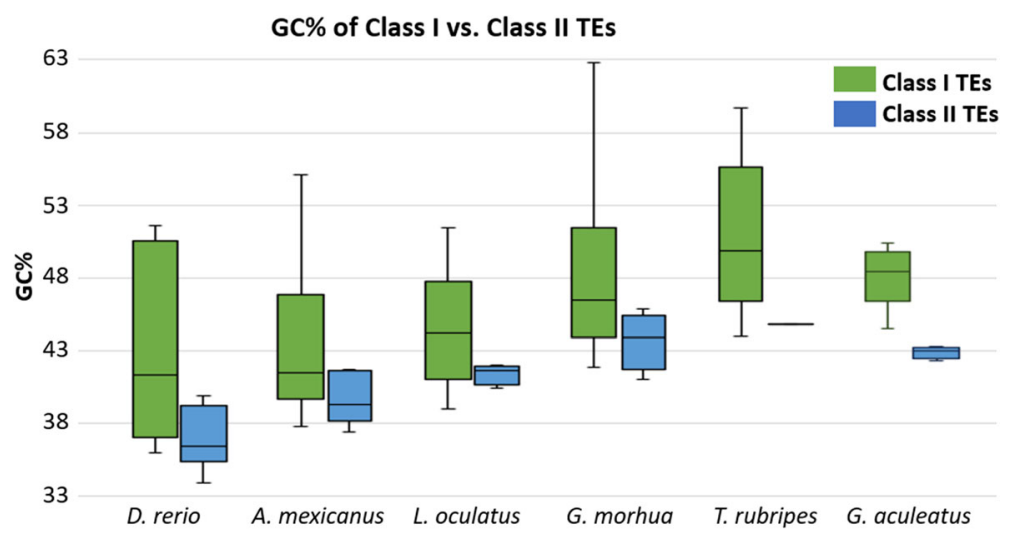

Fig. 4 Comparison GC\% between TE consensus sequences from Class I (retrotransposons) and Class II (DNA transposons) in six selected fish species (highlighted in the main text) listed in the FishTEDB database [34]

vertebrates has now become sufficient to begin to address the questions raised here.

\section{GC richness vs. AT/GC heterogeneity and TEs}

It is necessary to distinguish between an overall genomic GC-richness, i.e., $\mathrm{GC}_{\mathrm{G}}$, and the avian or mammalian situation of AT/GC heterogeneity (recorded also in nonteleost gars [36]). This entails an alternation of GC-rich and GC-poor regions along linkage groups, thus forming banding patterns on chromosomes upon an AT/GC-specific staining (recently reviewed by [36]). In the case of AT/GC heterogeneity, the overall $\mathrm{GC}_{\mathrm{G}}$ can be even lower than is in cases of AT/GC homogeneity typical for fish genomes as shown below. Considering that all of the currently available vertebrate genome assemblies contain gaps due to either repeat-rich or GC-rich regions [37], fish with GC-rich genomes might actually be even GCricher than currently estimated, and potentially even more GC-rich than mammalian and avian genomes. This is indicated by the following examples: the human $\left(\mathrm{GC}_{\mathrm{G}}=40.9 \%\right)$, mouse $\left(\mathrm{GC}_{\mathrm{G}}=42.5 \%\right)$, and even chicken $\left(\mathrm{GC}_{\mathrm{G}}=41.9 \%\right)$ genomes are $\mathrm{GC}$-poorer than $\operatorname{cod}\left(\mathrm{GC}_{\mathrm{G}}=\right.$ $46.3 \%)$ and three pufferfish species $\left(\mathrm{GC}_{\mathrm{G}}=45.6,45.7 \%\right.$ and $\mathrm{GC}_{\mathrm{G}}=46.6 \%$ respectively). However, note the situation in the non-teleost spotted gar with $\mathrm{GC}_{\mathrm{G}}=40.4 \%$ and AT/GC heterogeneity. The total length of its available assembly is merely $945.878 \mathrm{Mb}$ [33], which is remarkably incomplete in comparison with the cytological genome size estimate of $1.4 \mathrm{pg}$ [32]. Nevertheless, the AT/GC heterogeneity evidenced cytogenetically was also confirmed using genomic data [36].

The smaller and GC-rich(er) fish genomes also contain lower TE densities (or lower densities of GC-poor TEs) and/or GC-rich (er) TEs. The fact that the averaged $\mathrm{GC} \%$ of consensus sequences from all TE families is generally higher than the entire genomic GC\% suggests that $\mathrm{TE}$ spread and accumulation might contribute to the overall $\mathrm{GC}_{\mathrm{G}}$ in fish. This is further supported by our observation that genomes with a higher GC\% of the repetitive genomic fraction (i.e., TEs and other repeats; $\mathrm{GC}_{\mathrm{REP}}$ ) have a higher $\mathrm{GC}_{\mathrm{NONREP}}$, i.e., $\mathrm{GC} \%$ of the nonrepetitive rest of the genome. However, due to the broad range of $\mathrm{GC}_{\mathrm{TE}}$ of major groups of TEs in different species (Fig. 3), the activity and abundance of GC-poor(er) DNA transposons might also contribute to the AT/GC homogeneity in fish, assuming they accumulated more homogenously, compared to the AT/GC heterogeneity in avian and mammalian genomes that usually lack activity of DNA transposons.

\section{How could TEs shape the host nucleotide compositional landscape?}

Considering our findings, we anticipate at least three possible ways how TEs could influence the host nucleotide compositional landscape: 1) TEs shape it through inserting their "own" GC in a new context (i.e., increasing $\mathrm{GC} \%$ of the region if they have high GC; lowering GC\% of the region of they have low GC); 2) TEs shape nearby GC\% through "spillover" of CpG methylation ('sloping shores' model of [40]), leading to CpG hypermutation and thus decrease of nearby $\mathrm{GC} \%$; and 3 ) some TEs might contain sequence motifs that increase or decrease the local recombination landscape and thus the strength of GC-biased gene conversion. There are however many more questions about GC\% of TEs to be answered: Are quantitatively larger mobilomes as GCpoor as larger host genomes are overall? Why are DNA transposons GC-poor? Why are some DNA transposons $\mathrm{GC}$-poorer than others and only so in some species?

\section{Conclusion and perspectives}

Here we have shown that nucleotide composition of TEs and their interplay with host genomes is an unexplored part of genome biology. The GC-poor DNA transposons 
predominant in fish genomes and nearly absent in avian and mammalian genomes might have indeed contributed to shaping the nucleotide compositional landscape in vertebrates. Only the GC-heterogeneous gar and the GC-enriched pufferfishes possess GC-richer TEs and fewer DNA transposons. At the same time, among others the GC-poor genome of zebrafish possesses the GC-poorest TEs. Hence, it is possible that DNA transposon spreading and accumulation has actively contributed to the overall GC homogenization of fish genomes. On the other hand, replacement of DNA transposons by retrotransposons in avian and mammalian genomes might have contributed to their AT/GC heterogeneity through differential accumulation across chromosomes. The GC content of TEs should thus be considered as one of the factors potentially shaping the nucleotide compositional landscape in vertebrates and requires further investigations in detail. The next step envisaged is a qualitative analysis of the contribution $\mathrm{GC} \%$ of individual TE insertions to the GC\% of host genomes while accounting for TE copy number. This step can be combined with cytogenetic data to investigate the chromosomal distribution of various TEs and their potential contribution to the GC homogenization of fish genomes. With 55 fish species genome assemblies recently introduced by the 98th release of Ensembl (November 2019 [38]) and numerous others, such comprehensive analyses now appear feasible.

\section{Methods}

All species analysed in datasets produced for this study are listed in the Additional file 1: Table S1 and the datasets supporting the conclusions of this article are included in the Additional file 2: Table S2. We obtained genome size data as C-values from the www.genomesize.com database [32]. At this stage, diverse sources of datasets and databases (ref. [3], Animal Genome Size Database [32], GenBank [33], FishTEDB [34]) list different sets of fish species of which only some have been analysed for TEs. Assembly size data in Mb were obtained from the NCBI GenBank records of sequenced genomes [33]. Proportions of TEs in fish genomes were obtained from ref. [3] and compared with ref. [7]. Sequences of annotated fish TEs were obtained from Fish TE database http://www.fishtedb. org [34] and from the Repbase database at www.girinst. org [41]. Further data were extracted from literature as listed in the Additional file 2: Table S2. We used custom Python scripts to extract $\mathrm{GC}_{\mathrm{REP}}$ (repeats including lowcomplexity regions) of fish genomes in the Ensembl database (https://www.ensembl.org/ [38]) and compared to $\mathrm{GC} \%$ of the rest of the genome assembly $\left(\mathrm{GC}_{\text {NONREP }}\right)$, i.e. the non-repetitive fraction. The scripts are available at the GitHub repository https://github.com/bioinfohk/GC_TE/ blob/master/GC_softmasked_genomesFISH.ipynb.

\section{Supplementary information}

Supplementary information accompanies this paper at https://doi.org/10. 1186/s13100-019-0195-y.

Additional file 1: Table S1. Species overview and their counts.

Additional file 2: Table S2. Datasets used for generating Figs. 1, 2, 3, 4 and Additional files 3 and 4: Figures S1-S2.

Additional file 3: Figure S1. Analysis of genome size vs. $\mathrm{GC}_{\mathrm{G}}$ including salmonids (for comparison with Fig. 1b).

Additional file 4: Figure S2. Comparison of $\mathrm{GC}_{\mathrm{G}}$ and $\mathrm{GC}_{\mathrm{TE}}$ in 29 fish species (ray-finned fish and outgroups lancelet Branchiostoma belcheri, lamprey Petromyzon marinus, shark Callorhinchus milii, and coelacanth Latimeria chalumnae) listed in the FishTEDB [36]. In only two species analysed, $\mathrm{GC}_{\mathrm{TE}}$ (orange) is lower than $\mathrm{GC}_{\mathrm{G}}$ (blue; $A$. anguilla and $\mathrm{G}$. morhua). Based on the dataset for Fig. 1C in Additional file 2.

Additional file 5: Figure S3. Species-specific comparisons of $\mathrm{GC}_{\mathrm{TE}}$ between Class I and Class II TEs.

\section{Abbreviations}

GC\%: Percentage of $\mathrm{G}+\mathrm{C}$ bases, i.e., the molar ratio of guanine and cytosine in DNA; $\mathrm{GC}_{\mathrm{G}}$ : GC\% of the whole genome; $\mathrm{GC}_{\text {NONREP }}$ : GC\% of the nonrepetitive fraction of genome assemblies in Ensembl; $\mathrm{GC}_{\text {REP }}$ : $\mathrm{GC} \%$ of the repetitive fraction of genome assemblies in Ensembl; $\mathrm{GC}_{\mathrm{TE}}$ : GC\% of TE consensus sequences; GS: Genome size; LINE: Long interspersed element; LTR: Long terminal repeat; MLE: Mariner-like element; SINE: Short interspersed element; TE: Transposable element; WGD: Whole genome duplication

\section{Acknowledgements}

We would like to acknowledge Carina Mugal and Cedric Feschotte for insightful discussions, and Jesper Boman and Homa Papoli Yazdi for helpful comments on an earlier version of this manuscript. We also thank two anonymous reviewers for their constructive suggestions on this manuscript. Furthermore, we would like to acknowledge Dominik Matoulek for preparation of Python scripts for $\mathrm{GC}_{\text {REP }}$ and $\mathrm{GC}_{\text {NONREP }}$ analysis and Michal Dobrovolný for his help with species-specific GC\% analysis in fish from FishTEDB.

\section{Authors' contributions}

RS conceived the study, RS drafted the first version of the manuscript, RS and AS co-drafted subsequent versions of the manuscript, RS received funds for the study. Both authors read and approved the final manuscript.

\section{Funding}

The authors are grateful to the 'Excelence projekt PrF UHK 2209/2018' for the financial support.

\section{Availability of data and materials}

All data generated or analysed during this study are included in this published article and its supplementary information files.

Ethics approval and consent to participate

Not applicable.

\section{Consent for publication}

Not applicable.

\section{Competing interests}

The authors declare that they have no competing interests.

\section{Author details}

1Department of Biology, Faculty of Science, University of Hradec Králové, Hradec Králové, Czech Republic. 'Department of Ecology and Genetics Evolutionary Biology, Evolutionary Biology Centre (EBC), Science for Life Laboratory, Uppsala University, Uppsala, Sweden. ${ }^{3}$ Present address: Department of Organismal Biology - Systematic Biology, Evolutionary Biology Centre (EBC), Science for Life Laboratory, Uppsala University, Uppsala, Sweden. 
Received: 27 September 2019 Accepted: 5 December 2019

Published online: 12 December 2019

\section{References}

1. Li X-Q, Du D. Variation, evolution, and correlation analysis of $C+G$ content and genome or chromosome size in different kingdoms and phyla. Zhang Z, editor. PLoS ONE. 2014;9:e88339.

2. Bernardi G. Structural and evolutionary genomics natural selection in genome evolution. Amsterdam: Elsevier; 2005. Available from: http://cmich. idm.oclc.org/login?url=http://site.ebrary.com/lib/cmich/Doc?id=10138474. [cited 2018 Nov 4 ]

3. Canapa A, Barucca M, Biscotti MA, Forconi M, Olmo E. Transposons, genome size, and evolutionary insights in animals. Cytogenet Genome Res. 2015;147: 217-39

4. Chalopin D, Naville M, Plard F, Galiana D, Volff J-N. Comparative analysis of transposable elements highlights mobilome diversity and evolution in vertebrates. Genome Biol Evol. 2015;7:567-80.

5. Brynildsen W. Transposable elements in teleost fish: in silico exploration of TE activity, diversity and abundance across 74 teleost fish genomes: University Oslo; 2016. Available from: http://urn.nb.no/URN:NBN:no-55565

6. Shao F, Han M, Peng Z. Evolution and diversity of transposable elements in fish genomes. Sci Rep. 2019;9 Available from: http://www.nature.com/ articles/s41598-019-51888-1. [cited 2019 Nov 21]

7. Gao B, Shen D, Xue S, Chen C, Cui H, Song C. The contribution of transposable elements to size variations between four teleost genomes. Mob DNA. 2016:7 Available from: http://www.mobilednajournal.com/ content/7/1/4. [cited 2018 Mar 19].

8. Braasch I, Gehrke AR, Smith JJ, Kawasaki K, Manousaki T, Pasquier J, et al. The spotted gar genome illuminates vertebrate evolution and facilitates human-teleost comparisons. Nat Genet. 2016:48:427-37.

9. Volff J-N, Bouneau L, Ozouf-Costaz C, Fischer C. Diversity of retrotransposable elements in compact pufferfish genomes. Trends Genet 2003;19:674-8

10. Gao Y, Gao Q, Zhang H, Wang L, Zhang F, Yang C, et al. Draft sequencing and analysis of the genome of pufferfish Takifugu flavidus. DNA Res. 2014 21:627-37.

11. Dasilva C, Hadji H, Ozouf-Costaz C, Nicaud S, Jaillon O, Weissenbach J, et al. Remarkable compartmentalization of transposable elements and pseudogenes in the heterochromatin of the Tetraodon nigroviridis genome. Proc Natl Acad Sci. 2002;99:13636-41.

12. Neafsey DE. Genome size evolution in pufferfish: a comparative analysis of Diodontid and Tetraodontid pufferfish genomes. Genome Res. 2003;13:821-30.

13. Howe K, Clark MD, Torroja CF, Torrance J, Berthelot C, Muffato M, et al. The zebrafish reference genome sequence and its relationship to the human genome. Nature. 2013;496:498-503.

14. Tørresen OK, Star B, Jentoft S, Reinar WB, Grove H, Miller JR, et al. An improved genome assembly uncovers prolific tandem repeats in Atlantic cod. BMC Genomics. 2017;18 Available from: http://bmcgenomics. biomedcentral.com/articles/10.1186/s12864-016-3448-x. [cited 2018 Jan 18].

15. Sotero-Caio CG, Platt RN, Suh A, Ray DA. Evolution and diversity of transposable elements in vertebrate genomes. Genome Biol Evol. 2017; 9:161-77.

16. Pritham EJ. Transposable elements and factors influencing their success in eukaryotes. J Hered. 2009;100:648-55

17. Kapusta A, Suh A, Feschotte C. Dynamics of genome size evolution in birds and mammals. Proc Natl Acad Sci. 2017;114:E1460-9.

18. Fryxell KJ, Zuckerkandl E. Cytosine deamination plays a primary role in the evolution of mammalian isochores. Mol Biol Evol. 2000;17:1371-83.

19. Mugal CF, Weber CC, Ellegren H. GC-biased gene conversion links the recombination landscape and demography to genomic base composition: GC-biased gene conversion drives genomic base composition across a wide range of species. BioEssays. 2015;37:1317-26.

20. Kent TV, Uzunović J, Wright SI. Coevolution between transposable elements and recombination. Philos Trans R Soc B Biol Sci. 2017:372:20160458.

21. Baker Z, Schumer M, Haba Y, Bashkirova L, Holland C, Rosenthal GG, et al. Repeated losses of PRDM9-directed recombination despite the conservation of PRDM9 across vertebrates. eLife. 2017;6 Available from: https:// elifesciences.org/articles/24133. [cited 2018 Nov 4].

22. Duret L, Hurst LD. The elevated GC content at exonic third sites is not evidence against neutralist models of isochore evolution. Mol Biol Evol. 2001;18:757-62
23. Lander ES, Linton LM, Birren B, Nusbaum C, Zody MC, Baldwin J, et al. Initial sequencing and analysis of the human genome. Nature. 2001;409:860-921.

24. Duret L, Mouchiroud D, Gautier C. Statistical analysis of vertebrate sequences reveals that long genes are scarce in GC-rich isochores. J Mol Evol. 1995;40:308-17.

25. Smit AF. Interspersed repeats and other mementos of transposable elements in mammalian genomes. Curr Opin Genet Dev. 1999;9:657-63.

26. Melodelima C, Gautier C. The GC-heterogeneity of teleost fishes. BMC Genomics. 2008:9:632

27. Halaimia-Toumi N, Casse N, Demattei MV, Renault S, Pradier E, Bigot Y, et al. The GC-rich transposon bytmar1 from the deep-sea hydrothermal crab, bythograea thermydron, may encode three transposase isoforms from a single ORF. J Mol Evol. 2004;59:747-60.

28. Casse N, Bui QT, Nicolas V, Renault S, Bigot Y, Laulier M. Species sympatry and horizontal transfers of mariner transposons in marine crustacean genomes. Mol Phylogenet Evol. 2006;40:609-19.

29. Bui Q-T, Delaurière L, Casse N, Nicolas V, Laulier M, Chénais B. Molecular characterization and phylogenetic position of a new mariner-like element in the coastal crab, Pachygrapsus marmoratus. Gene. 2007;396:248-56.

30. Ferguson AA, Jiang N. Pack-MULEs: recycling and reshaping genes through GC-biased acquisition. Mob Genet Elem. 2011;1:135-8.

31. Dion-Côté A-M, Symonová R, Lamaze FC, Pelikánová Š, Ráb P, Bernatchez L. Standing chromosomal variation in Lake whitefish species pairs: the role of historical contingency and relevance for speciation. Mol Ecol. 2017;26:178-92.

32. Gregory TR. Animal genome size database. http://www.genomesize.com.

33. Benson DA, Cavanaugh M, Clark K, Karsch-Mizrachi I, Lipman DJ, Ostell J, et al. GenBank. Nucleic Acids Res. 2013;41:D36-42.

34. Shao F, Wang J, Xu H, Peng Z. FishTEDB: a collective database of transposable elements identified in the complete genomes of fish. Database. 2018;2018. https://doi.org/10.1093/database/bax106.

35. Macqueen DJ, Johnston IA. A well-constrained estimate for the timing of the salmonid whole genome duplication reveals major decoupling from species diversification. Proc R Soc B Biol Sci. 2014;281:20132881.

36. Symonová R, Majtánová Z, Arias-Rodriquez L, Mořkovský L, Kořínková T, Cavin L, et al. Genome compositional organization in gars shows more similarities to mammals than to other ray-finned fish: cytogenomics of gars. J Exp Zool B Mol Dev Evol. 2017;328:607-19.

37. Peona $\mathrm{V}$, Weissensteiner $\mathrm{MH}$, Suh $\mathrm{A}$. How complete are "complete" genome assemblies?-an avian perspective. Mol Ecol Resour. 2018;18:1188-95.

38. Zerbino DR, Achuthan P, Akanni W, Amode MR, Barrell D, Bhai J, et al. Ensembl 2018. Nucleic Acids Res. 2018:46:D754-61.

39. Brynildsen WR. Transposable elements in teleost fish - in silico explorations of TE activity, diversity and abundance across 74 teleost fish genomes. 2016 Available from: https://www.duo.uio.no/handle/10852/52365

40. Grandi FC, Rosser JM, Newkirk SJ, Yin J, Jiang X, Xing Z, et al. Retrotransposition creates sloping shores: a graded influence of hypomethylated CpG islands on flanking CpG sites. Genome Res. 2015;25: 1135-46.

41. Bao W, Kojima KK, Kohany O. Repbase update, a database of repetitive elements in eukaryotic genomes. Mob DNA. 2015;6 Available from: http:// www.mobilednajournal.com/content/6/1/11. [cited 2018 Nov 4].

\section{Publisher's Note}

Springer Nature remains neutral with regard to jurisdictional claims in published maps and institutional affiliations.

Ready to submit your research? Choose BMC and benefit from:

- fast, convenient online submission

- thorough peer review by experienced researchers in your field

- rapid publication on acceptance

- support for research data, including large and complex data types

- gold Open Access which fosters wider collaboration and increased citations

- maximum visibility for your research: over $100 \mathrm{M}$ website views per year

At $\mathrm{BMC}$, research is always in progress.

Learn more biomedcentral.com/submissions 